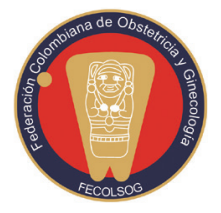

Revista Colombiana de Obstetricia y Ginecología Vol. 65 No. 2 • Abril-Junio 2014 • (129-138)

\title{
ACUERDO ENTRE TRES EVALUADORES Y UN EXPERTO EN LA DETECCIÓN DE RIESGO RELACIONAL EN EL POSPARTO ENTRE LA MADRE Y SU RECIÉN NACIDO, TEMUCO, IX REGIÓN, CHILE, 2010
}

\section{Agreement among three examiners and one expert in the detection of mother-infant attachment risk during the post-partum period, Temuco, IX región, Chille, 2010}

\begin{abstract}
Maribel Muñoz-Molina, MSc'; Ana María Poo-Figueroa, MSc'; Luis Bustos-Medina ${ }^{3}$; Bernardita Baeza-Weinmann, $\mathrm{MSc}^{4}$
\end{abstract}

Recibido: diciembre 12/13 - Aceptado: junio 20/14

\section{RESUMEN}

Introducción: después del nacimiento es recomendable evaluar el vínculo entre la madre y el recién nacido para detectar riesgo de alteraciones en esta interacción y planificar intervenciones precoces para favorecer el apego seguro. El evaluador debe realizar observaciones confiables para el diagnóstico, para lo cual requiere capacitación, entrenamiento y experiencia.

1 Magíster en Estudios Psicológicos. Profesor Asistente, Departamento de Pediatría y Cirugía Infantil. Docente Carrera de Obstetricia y

Puericultura. Especialidad en Perinatología, Universidad de La Frontera, Temuco, Chile.

2 Magíster en Pedagogía y Gestión Universitaria. Profesor Asistente, Departamento de Psicología, Facultad de Educación, Ciencias Sociales y Humanidades. Docente Carrera de Obstetricia y Puericultura, Especialidad en Perinatología, Universidad de La Frontera, Temuco, Chile.

3 Licenciado en Educación Matemática, Bioestadístico. Profesor Asistente, Departamento de Salud Pública, CIGES, Facultad de Medicina, Universidad de La Frontera, Temuco, Chile.

4 Magíster en Epidemiología Clínica. Profesor Asistente, Departamento de Pediatría y Cirugía Infantil. Docente Carrera de Obstetricia y Puericultura, Especialidad en Perinatología, CIGES, Facultad de Medicina, Universidad de La Frontera, Temuco, Chile. bernardita.baeza@ufrontera.cl
Objetivo: describir la concordancia interobservador entre tres evaluadores y un experto en la detección de riesgo relacional entre la madre y su recién nacido, aplicando la pauta de evaluación relacional de Kimelman.

Materiales y métodos: estudio de corte transversal. Se evaluaron diadas madre-hijo durante su estadía en el Hospital Hernán Henríquez Aravena de Temuco, centro de referencia de la región de la Araucanía, sur de Chile. Se excluyeron las madres de recién nacidos hospitalizados. Muestra no probabilística por conveniencia. La concordancia interobservador fue medida en el diagnóstico del riesgo de mala interacción y en las variables de la pauta relacional de Kimelman. Para el análisis se utilizó la estadística kappa, se consideraron aceptables las observaciones interobservador con valor kappa mayor a 0,5.

Resultados: se evaluaron 71 díadas madre-recién nacido (M-RN) en el periodo comprendido entre el 1 y 30 de marzo de 2010. La concordancia en los 
ítems que evalúan la interacción entre la madre y el recién nacido se encuentra entre 0 y 1 . En los ítems que evalúan el grado de aceptación la concordancia está entre 0,59 y 0,86. La calificación de la armonía de la interacción está sobre 0,57 ; la tonalidad afectiva entre 0,42 y 0,83; el diagnóstico, tanto del alto riesgo como del bajo riesgo relacional, fluctúa entre 0,43 y 0,91 .

Conclusión: se enfatiza la importancia de la capacitación con base en la teoría del apego, las características de la interacción diádica y el desarrollo de competencias para entrevistar y observar sistemáticamente, a fin de obtener certeza de las conductas observadas.

Palabras clave: apego, puerperio, recién nacido.

\section{ABSTRACT}

Introduction: It is advisable to assess the bonding between the mother and the newborn in order to identify abnormalities in this interaction and plan for early interventions to facilitate attachment. The examiner must make reliable observations for diagnosis and this requires training and experience. Objective: To describe inter-observer agreement among three examiners and one expert in the detection of mother-infant attachment risk between, applying the Kimelman attachment assessment guide.

Materials and methods: Cross-sectional study. Mother-infant pairs were assessed during their stay at Hospital Hernán Henríquez Aravena in Temuco, a referral centre for the Araucanía region in southern Chile. Mothers of hospitalized infants were excluded. A non-probabilistic sample was used for convenience. Inter-observer agreement was measured in relation to the diagnosis of the risk of poor interaction and the variables of the Kimelman attachment guide. Kappa statistics was used for the analysis and inter-observer observations were considered acceptable when the kappa value was greater than 0.5 .

Results: Overall, 71 mother-infant (M-I) pairs were assessed during the period between March $1^{\text {st }}$ and
March $30^{\text {th }}, 2010$. Agreement regarding the items that assess the interaction between the mother and the newborn is in the range of 0 to 1 . Items that assess the degree of acceptance are between 0.59 and 0.86 . The rating for interaction harmony is above 0.57 ; affective tone is between 0.42 and 0.83 ; the diagnosis of high as well as low relational risk ranges between 0.43 and 0.91 .

Conclusion: We emphasize the importance of training based on the theory of attachment, the characteristics of the dyadic interaction, and the development of interviewing and systematic observation competencies in order to be certain about observed behaviours.

Key words: Attachment, post-partum, newborn.

\section{INTRODUCCIÓN}

En Chile, desde el año 2008, el Ministerio de Salud implementa como política pública de salud el sistema de protección integral a la primera infancia, "Chile crece contigo" (1). Este incorpora en sus programas la atención personalizada del proceso reproductivo desde sus inicios, incluyendo la evaluación del vínculo de apego entre la madre y su recién nacido $(\mathrm{RN})$ con el fin de detectar el riesgo en esta interacción y establecer acciones que favorezcan dicho vínculo (1).

En los seres humanos, el tipo de vínculo de apego que se consolida hacia el final del primer año se adquiere a través de la interacción y, en consecuencia, se modula por variables psicológicas y ambientales (2). La madre es quien deberá poseer la sensibilidad para interpretar las señales del bebé a fin de responder afectiva y conductualmente de manera apropiada y rápida, estableciendo una interacción armónica donde las competencias sensoriales e interactivas del bebé y las competencias maternales se acoplen sintónicamente (3-5). Este tipo de relación, definido como vínculo de apego seguro, tiene como fin la búsqueda de proximidad con la figura significativa de apego que le permita al bebé sentirse protegido frente a situaciones de angustia, y se constituye en la base para el desarro- 
llo psicoafectivo normal del niño. Sin embargo, es posible observar otras formas de interacción que generan trastornos vinculares, tales como vínculo de apego ansioso evitativo, ansioso ambivalente y desorganizado (3, 5-8).

Entre los factores que dificultan establecer un vínculo de apego seguro se describen: la ausencia de la madre o sustituto maternal, discontinuidad de la relación con la figura vincular y trastornos cuantitativos de la interacción dados por hiperestimulación o hipoestimulación $(4,5)$. Otros autores plantean que el vínculo de apego puede alterarse cuando el cuidador fracasa en leer los estados mentales o las necesidades del bebé, ello puede ocurrir en madres con depresión perinatal, duelo no resuelto, pérdidas neonatales previas, anomalías fetales, conflictos de pareja, entre otros (9-14). Respecto a las variables del niño se describen: antecedentes de prematurez, enfermedad crónica y malformaciones $(3,4)$, y entre las variables socioculturales: madre adolescente, monoparentalidad y analfabetismo $(2-4,7)$.

Evaluar la díada madre-recién nacido (M-RN) en su estadía hospitalaria posparto es importante pues permite detectar precozmente el riesgo relacional e intervenir para prevenir sus consecuencias. Para que la evaluación sea confiable, el equipo perinatal debe entrenarse en técnicas de observación de la díada y entrevista a la madre dado que, según señala Kimelman, "la observación de la interacción entre el bebé y la madre tiene un componente concreto evidenciado a través de los diálogos visuales, tónicos y auditivos y un componente subjetivo, imaginario que constituye la trama de la relación"; también plantea que para comprender una disfunción de la interacción madre-bebé se debe decodificar la articulación entre los componentes concreto e imaginario de la relación (4).

Dado que la evaluación relacional para detectar trastornos del vínculo de apego utiliza como herramienta principal la técnica observacional y la entrevista, es relevante considerar que estas pueden presentar variaciones entre los evaluadores. La observación de acontecimientos vitales intensos como el parto y el puerperio es una vía de acceso al conocimiento de todas las experiencias que repercuten sobre la subjetividad $(15,16)$. Una de las directrices recomendadas para mejorar la validez de los datos observacionales es entrenar a los observadores para que sean tan objetivos como sea posible y puedan separar la observación de la interpretación, así como observar un número limitado de conductas específicas definidas a priori. El empleo de varios observadores y la obtención de una muestra representativa de observaciones también pueden mejorar la precisión de estas. Aiken señala que debe entrenarse a los observadores en qué buscar y cómo registrar sus observaciones, cómo distinguir entre lo que se observa y la manera en que se interpreta (15).

La entrevista como herramienta psicológica comparte con los métodos de observación los problemas de confiabilidad y validez. La confiabilidad requiere consistencia, pero los entrevistadores varían en su apariencia, enfoque, estilo y, en consecuencia, en la impresión que causan en los entrevistados. La confiabilidad de una entrevista se determina comparando las calificaciones dadas a las respuestas del entrevistador por dos o más jueces. Además, esta puede aumentarse con el entrenamiento minucioso de los entrevistadores $(17,18)$. Respecto de la entrevista a los padres, Kimelman señala que estos informan del comportamiento y los síntomas de su hijo en forma subjetiva, que sus verbalizaciones acerca de los sentimientos, pensamientos y expectativas en relación con este y el modo en que perciben sus competencias como padres deben ser acogidas activamente y confrontadas con la observación "objetiva” del hijo. En cuanto a la actitud del profesional que realiza la evaluación, Kimelman señala que debe "propiciar el relato verbal espontáneo y debe mantener una visión bifocal dirigida tanto a las interacciones padres-bebé como al relato verbal de los padres" (4).

El presente artículo tiene como objetivo establecer la concordancia entre tres evaluadores y un experto en la detección de riesgo relacional en la madre y su recién nacido, aplicando la pauta de evaluación relacional madre-bebé de Kimelman (6). 


\section{MATERIALES Y MÉTODOS}

Estudio de corte transversal en el que se evaluaron díadas madre-recién nacido (M-RN) atendidas en la maternidad del hospital público Dr. Hernán Henríquez Aravena, centro de alta complejidad y de referencia de la región de la Araucanía de Temuco, Chile. Se incluyeron madres de RN a término, sanos, de parto normal o cesárea; se excluyeron las madres que manifestaron dar su hijo en adopción y madres con patología que impidiera ser la cuidadora principal de su hijo. La muestra fue no probabilística por conveniencia con un total de 71 diadas.

Procedimiento. Las madres se entrevistaron antes del tercer día posparto en la sala de puerperio en donde permanecían en alojamiento conjunto con su hijo RN. Los evaluadores correspondieron a una experta -psicóloga clínica con amplia experiencia en evaluación de riesgo relacional-y tres profesionales de las cuales dos eran matronas especialistas en neonatología (evaluadoras 2 y 3 ) y una psicólogamatrona (evaluadora 4). Las evaluadoras 2, 3 y 4 fueron capacitadas por expertas de la Universidad de Chile en el uso del instrumento de evaluación, un mes previo al estudio. La capacitación incluyó los siguientes aspectos teóricos: competencias parentales, teoría del apego y características de la interacción diádica. Para los aspectos prácticos los expertos aplicaron la pauta a díadas M-RN, efectuando registro audiovisual durante el proceso; posteriormente se realizó análisis crítico de los videos para corregir las técnicas de entrevista y de observación conductual.

Después de esta capacitación, cada evaluadora valoró díadas simultáneamente con un juez experto, realizando los registros en forma independiente. De esta manera se obtuvieron dos evaluaciones por cada díada en un mismo momento.

Como instrumento de evaluación se utilizó la pauta relacional de Kimelman, creada en 1995 y validada en $5.600 \mathrm{M}-\mathrm{RN}$ chilenos. Consiste en 15 ítems que evalúan conductas y actitudes maternales indicadoras del proceso de apego (anexo). Las conductas por evaluar consisten en comportamientos que presentan los recién nacidos y sus madres y que dan cuenta de la interacción M-RN (5). Este instrumento contiene ítems de evaluación global de riesgo con correlación satisfactoria (coeficiente de correlación de Spearman entre 0,3 y 0,738), confiabilidad (alfa de Cronbach 0,88 ) y concordancia en el diagnóstico de riesgo relacional de 94,3\%. Esta pauta finaliza con la evaluación global de: "alto riesgo" o "bajo riesgo" de alteración del vínculo de apego, ya que en este proceso de vinculación la existencia del riesgo está siempre presente.

La concordancia interobservador fue medida en el diagnóstico obtenido (alto riesgo o bajo riesgo) como también en todas las variables que contempla la pauta relacional: observación de la interacción entre la madre y el recién nacido (contacto visual, contacto físico y ocurrencia de verbalización), grado de aceptación del sexo y del aspecto físico; además se evaluó el tipo de interacción (armónico o disarmónico) y la tonalidad afectiva utilizada por la madre en la interacción con su recién nacido.

Para el análisis de los datos se utilizó la estadística kappa, dadas las recomendaciones actuales (19) en la aplicación de esta estadística en medición de eventos clínicos. Para esta investigación se confeccionó una tabla de valores que consideró aceptables las observaciones interjuez que arrojaron un valor kappa mayor a 0,5. El software utilizado para la entrada de datos y análisis fue Stata 9.0.

Este estudio contó con la aprobación del Comité de Ética del servicio de salud, el permiso de las autoridades de la maternidad y el patrocinio de la Universidad de La Frontera. Se contó con el consentimiento informado de todas las madres participantes del estudio. Posterior a la aplicación de la pauta, los casos detectados en crisis fueron remitidos a la psicóloga de la maternidad para apoyo inmediato.

\section{RESULTADOS}

Se evaluaron 71 díadas M-RN en el periodo comprendido entre el 1 y 30 de marzo de 2010. Todas las participantes eran beneficiarias del sistema 
público de salud, 55 pertenecientes al nivel socioeconómico bajo; 22 de ellas solteras o separadas, 15 casadas, 34 convivientes; 13 estaban a cargo de la crianza de los hijos sin apoyo de su pareja; 18 con trabajo remunerado; 56 madres entre 20 y 30 años, 14 adolescentes y una mayor de 40 años; 45 tuvieron parto vaginal y 26 por cesárea; 62 díadas M-RN tuvieron contacto piel a piel en el posparto inmediato. Todas las madres se encontraban en condiciones de amamantar y brindar cuidados a su $\mathrm{RN}$ al momento de la entrevista.

El experto (psicólogo) evaluó $71 \mathrm{M}-\mathrm{RN}$ en total; la segunda evaluadora, $22 \mathrm{M}-\mathrm{RN}$; la tercera evaluadora, 24 M-RN, y la cuarta evaluadora, $25 \mathrm{M}-\mathrm{RN}$.

Los ítems que arrojaron mayor concordancia fueron: contacto visual, ocurrencia de verbalización y ocurrencia del contacto físico en la interacción M-RN. Los ítems aceptación del sexo y del aspecto físico, y asignación del nombre del bebé obtuvieron una alta concordancia. En algunos ítems -aceptación del sexo y del aspecto físico-los evaluadores no presentaron variación en sus respuestas. En relación con la asignación del nombre del recién nacido, tanto el evaluador 2 como el juez respondieron que sí en el 100\% de las observaciones (tabla 1). El observador 3 presenta valores de concordancia limítrofes o inferiores a kappa de 0,5 para el ítem de tonalidad afectiva.

Respecto a la tolerancia de las madres frente al llanto del hijo se presenta concordancia en los ítems que evalúan si la madre intenta calmarlo o si se aleja del RN (tabla 2), excepto para el evaluador 3 quien presenta respuestas contrarias y, por tanto, un kappa negativo.

Finalmente se presenta con alta concordancia la conclusión diagnóstica tanto del alto como del bajo riesgo relacional (tabla 3) para los evaluadores 2 y 4 , mientras que el evaluador 3 obtuvo un coeficiente de acuerdo inferior a 0,5 .

\section{DISCUSIÓN}

Los ítems evaluados a través de la observación obtuvieron mayor concordancia considerando, de acuerdo con Kimelman, que los aspectos concretos de la relación diádica serían más fáciles de percibir e interpretar que los aspectos subjetivos del componente imaginario (6).

Los ítems evaluados con la entrevista tienen menor acuerdo, como en el caso de la evaluación de la irritación de la madre frente al llanto, ya que la entrevista es un relato espontáneo y abierto. La interpretación realizada por el evaluador puede variar existiendo la posibilidad de que el evaluador interprete erróneamente la información que entrega la madre, de ahí la importancia, como señala la autora, de confrontar este relato con la observación objetiva de la interacción diádica (6).

De acuerdo con Aiken (15), en este estudio las investigadoras enfatizan que a través de la observación sistemática se debe obtener certeza de las conductas observadas para lo cual, si es necesario, debe reevaluarse a la díada dada la necesidad de lograr la apertura de la madre. De este modo se reducen las barreras que dificultan la entrega de un relato sincero de sus emociones, deseos, y expectativas en torno a la experiencia del embarazo y el entorno psicosocial que vivencia. Esta apertura implica tiempo del evaluador, que debe reiterar la observación hasta obtener una visión global de la interacción diádica.

Los evaluadores deben contar con capacitación en teoría del apego, características de la interacción diádica y desarrollar competencias para entrevistar, buscando en forma dirigida el componente subjetivo, dadas las características del instrumento que implica manejo de observación clínica, por ejemplo, estudiar la conducta no verbal (movimientos corporales), la conducta espacial (distancia espacial interpersonal en la comunicación), la conducta extralingüística (tono de voz, rapidez, secuencia en las intervenciones) y la conducta lingüística. Según lo planteado por Kimelman, la bifocalidad debe explicitarse y entrenarse en los evaluadores, ya que esta presenta dificultades, especialmente para aquellas observaciones que deben correlacionar el reporte oral de la madre con su estado afectivo 


\begin{tabular}{|c|c|c|c|c|}
\hline Criterio & Acuerdo observado & Acuerdo esperado & Kappa & $\mathbf{P}$ \\
\hline \multicolumn{5}{|l|}{ Interacción armónica } \\
\hline Acuerdo entre evaluadores 1 y 2 & 85,71 & 63,31 & 0,5882 & 0,0015 \\
\hline Acuerdo entre evaluadores 1 y 3 & 82,61 & 59,92 & 0,5660 & 0,0013 \\
\hline Acuerdo entre evaluadores 1 y 4 & 100 & 51,39 & 1 & $<0,0001$ \\
\hline \multicolumn{5}{|l|}{ Tonalidad afectiva } \\
\hline Acuerdo entre evaluadores 1 y 2 & 85,71 & 51,7 & 0,7042 & 0,0006 \\
\hline Acuerdo entre evaluadores 1 y 3 & 70,83 & 50,0 & 0,4167 & 0,0060 \\
\hline Acuerdo entre evaluadores 1 y 4 & 92,00 & 51,68 & 0,8344 & $<0,0001$ \\
\hline \multicolumn{5}{|l|}{ Contacto físico } \\
\hline Acuerdo entre evaluadores 1 y 2 & 90,48 & 68,71 & 0,6957 & 0,0004 \\
\hline Acuerdo entre evaluadores 1 y 3 & 88,00 & 75,84 & 0,5033 & 0,0054 \\
\hline Acuerdo entre evaluadores 1 y 4 & 96,00 & 70,40 & 0,8649 & $<0,0001$ \\
\hline \multicolumn{5}{|l|}{ Contacto visual } \\
\hline Acuerdo entre evaluadores 1 y 2 & 95,00 & 86,00 & 0,6429 & 0,0010 \\
\hline Acuerdo entre evaluadores 1 y 3 & 100 & 84,12 & 1 & $<0,0001$ \\
\hline Acuerdo entre evaluadores 1 y 4 & 95,65 & 74,10 & 0,831 & $<0,0001$ \\
\hline \multicolumn{5}{|l|}{ Ocurrencia de verbalización } \\
\hline Acuerdo entre evaluadores 1 y 2 & 90,48 & 75,06 & 0,6182 & 0,0011 \\
\hline Acuerdo entre evaluadores 1 y 3 & 87,50 & 64,58 & 0,6471 & 0,0007 \\
\hline Acuerdo entre evaluadores 1 y 4 & 95,83 & 60,42 & 0,8947 & $<0,0001$ \\
\hline \multicolumn{5}{|c|}{ Grado de aceptación de las características del recién nacido } \\
\hline \multicolumn{5}{|c|}{\begin{tabular}{|l|l|l|} 
Aceptación del sexo & & \\
\end{tabular}} \\
\hline Acuerdo entre evaluadores 1 y 2 & 90,48 & 90,48 & 0,0000 & - \\
\hline Acuerdo entre evaluadores 1 y 3 & 96,00 & 70,40 & 0,8649 & $<0,0001$ \\
\hline Acuerdo entre evaluadores 1 y 4 & 96,00 & 75,84 & 0,8344 & $<0,0001$ \\
\hline \multicolumn{5}{|l|}{ Aceptación del aspecto físico } \\
\hline Acuerdo entre evaluadores 1 y 2 & 95,24 & 95,24 & 0,0000 & - \\
\hline Acuerdo entre evaluadores 1 y 3 & 96,00 & 96,00 & 0,0000 & - \\
\hline Acuerdo entre evaluadores 1 y 4 & 92,00 & 78,56 & 0,6269 & 0,0004 \\
\hline \multicolumn{5}{|l|}{ Asignación del nombre } \\
\hline Acuerdo entre evaluadores 1 y 2 & 100 & - & - & - \\
\hline Acuerdo entre evaluadores 1 y 3 & 95,83 & 88,19 & 0,6471 & 0,0004 \\
\hline Acuerdo entre evaluadores 1 y 4 & 96,00 & 88,64 & 0,6479 & 0,0003 \\
\hline
\end{tabular}

*Evaluador 1: experto; evaluador 2: matrona; evaluador 3: matrona; evaluador 4: psicóloga y matrona. 


\begin{tabular}{|l|c|c|c|}
\hline \multicolumn{2}{|c|}{ Tabla 2. } \\
\hline \multicolumn{1}{|c|}{ Concordancia entre experto y evaluadores 2, 3 y 4 en la evaluación de la tolerancia } \\
de las madres frente al llanto del recién nacido*
\end{tabular}

${ }^{*}$ Evaluador 1: experto; evaluador 2: matrona; evaluador 3: matrona; evaluador 4: psicóloga y matrona.

\section{Tabla 3.}

Concordancia entre experto y evaluadores, del nivel de riesgo de alteración en la vinculación madre-recién nacido

\begin{tabular}{|l|c|c|c|c|}
\hline Evaluador & Acuerdo observado & Acuerdo esperado & Kappa & P \\
\hline Riesgo bajo & & & 0,6000 & 0,0025 \\
\hline Acuerdo entre evaluadores 1 y 2 & 80,95 & 52,38 & 0,04262 & 0,0161 \\
\hline Acuerdo entre evaluadores 1 y & 72,00 & 51,20 & 0,9110 & $<0,0001$ \\
\hline Acuerdo entre evaluadores 1 y 4 & 96,00 & 55,04 & 0,6000 & 0,0025 \\
\hline Riesgo alto & & & 0,4262 & 0,0162 \\
\hline Acuerdo entre evaluadores 1 y 2 & 80,95 & 52,38 & 0,9110 & $<0,0001$ \\
\hline Acuerdo entre evaluadores 1 y 3 & 72,00 & 51,20 & 55,04 & \\
\hline Acuerdo entre evaluadores 1 y 4 & 96,00 & &
\end{tabular}

\footnotetext{
* Evaluador 1: experto; evaluador 2: matrona; evaluador 3: matrona; evaluador 4: psicóloga y matrona.
} 
general y el estado de bienestar del recién nacido (4). Cuando el evaluador persiste con dudas respecto de sus observaciones, dificultando el diagnóstico, debe contar con el apoyo de un segundo evaluador debido a las implicancias de una derivación errónea.

Los autores identifican como una debilidad de este trabajo el no contar con evaluaciones previas a la capacitación. Esto habría permitido determinar la variación esperada en la concordancia de las observaciones interjuez posterior a este aprendizaje, visualizada como relevante para reforzar la recomendación de necesidad de capacitación de los profesionales que deben realizar el diagnóstico de la relación vincular de apego en este periodo de la vida.

\section{CONCLUSIÓN}

Los resultados presentados en este artículo permitieron a las investigadoras fundamentar la importancia de la capacitación en la aplicación de la pauta de riesgo relacional. Especialmente se refuerza la necesidad de ejercitar, discutir y analizar las técnicas de observación y entrevistas aplicadas de forma sistemática.

\section{FUENTE DE FINANCIACIÓN}

Proyectos de Investigación Científica (Diufro). Proyecto DI08-0057, Universidad de La Frontera, Temuco, Chile.

\section{REFERENCIAS}

1. Ministerio de Salud. Manual de atención personalizada en el proceso reproductivo. Santiago: Gobierno de Chile; 2014.

2. Bowlby J. Una base segura. Aplicaciones clínicas de la teoría del apego. Barcelona: Paidós; 1995.

3. Kimelman M, González L. El proceso de vinculación, una tarea del equipo obstétrico. Rev. Interactiva Foro Ginecológico 2003;6:10-13.

4. Kimelman M. Psicopatología del Bebé. En: Montenegro H, Guajardo H, editores. Psiquiatría del Niño y del Adolescente. 2 ed. Santiago: Mediterráneo; 2000. p. 337-432.
5. Barudy J, Marquebreucq A. Hijos e hijas de madres resilientes. Madrid: Gedisa; 2006.

6. Kimelman M, Núñez C, Hernández G, Castillo N, Páez J, Bustos S, et al. Construcción y evaluación de pauta de riesgo relacional madre recién nacido. Rev. Médica Chile. 1995;123:707-12.

7. Bowlby J. Vínculos afectivos: formación, desarrollo y pérdida. Madrid: Morata; 2003.

8. López F. El Apego. En: Palacios J, Marchesi A, Carretero M, editores. Psicología evolutiva. Desarrollo cognitivo y social del niño. Madrid: Alianza Editorial; 1999. p. 105-43.

9. Lecannelier F. La entrevista de apego de niños. Rev. Terapia Psicológica. 2002;20:50-3.

10. Jadresic E, Araya R, Jara, C. Validation of the Edinburgh Postnatal Depression Scale (EPDS) in Chilean postpartum women. Journal Psychosomatic Obstetric Gynecologic. 1995;16:187-91 [visitado 2014 mayo 22]. Disponible en: http://www.ncbi.nlm. nih.gov/pubmed/8748993.

11. Lecannelier F. Apego e intersubjetividad: influencia de los vínculos tempranos en el desarrollo humano y la salud mental. Santiago:LOM; 2006. p. 35-9.

12. Motta M, Lucion A, Manfro G. Efectos de la depresión materna en el desarrollo neurobiológico y psicológico del niño. Revista de Psiquiatría de Río Grande do Sul. 2005;27(2):165-76 [visitado 2014 mayo 22]. Disponible en: http://www.scielo.br/scielo.php?pid=S0101$81082005000200007 \& \operatorname{Ing}=\mathrm{en} \& \mathrm{nrm}=$ iso

13. Risco L, Jadresic E, Galleguillos T. Depresión posparto: alta frecuencia en puérperas chilenas. Detección precoz, seguimiento y factores de riesgo. Revista Psiquiatría y Salud Integral. 2002;2:61-6.

14. Perry B, Polard R. Homeostasis, stress, trauma and adaptation. A Neurodevelopmental view of childhood trauma. Child and adolescent psychiatric clinics of North America. 1998;7:33-51.

15. Aiken L. Test psicológicos y evaluación, 11 ed. México: Pearson; 2003. p. 85-94.

16. Mora A. El cuerpo investigador, el cuerpo investigado. Una aproximación fenomenológica a la experiencia del puerperio. Revista Colombiana de Antropología. 2009;45:11-38. 
17. Borman WC, Hanson M, Hedge J. Personnel Selection. Annual Review of Psichology. 1997;48: 299-337.

18. Campion M, Pursell E, Brown B. Structure interviewing: Raising the psychometric properties of the employment interview. Personnel Psychology. 1988;41:25-42.
19. Cortés E, Rubio JA, Gaitán H. Métodos estadísticos de evaluación de la concordancia y la reproducibilidad de pruebas diagnósticas. Rev Colomb Obstet Ginecol. 2010;61:247-55.

\section{Conflicto de intereses: ninguno declarado.}

